www.jmscr.igmpublication.org

Impact Factor 5.84

Index Copernicus Value: 83.27

ISSN (e)-2347-176x ISSN (p) 2455-0450

crossref DOI: _https://dx.doi.org/10.18535/jmscr/v5i4.161

Journal Of Medical Science And Clinical Research

\title{
A Study on Cardiac Manifestations of Dengue Fever
}

\author{
Authors \\ Abhinandya Mukhopadhyay ${ }^{1}$, Rakesh Kumar ${ }^{2}$, Dr B.K.Singh ${ }^{3}$ \\ ${ }^{1,2}$ MBBS, Junior Resident, General Medicine, Darbhanga Medical College, Bihar \\ ${ }^{3}$ Head of the Department, Professor, Department of General Medicine, DMC, Bihar
}

\begin{abstract}
Aims and Objectives: To study the cardiac manifestations of the dengue fever.

Method: This two year descriptive study was undertaken at Darbhanga Medical College and Hospital, department of medicine, Darbhanga, Bihar. 50 patients admitted with dengue NS1 Ag, dengue IgM or IgM and IgG positivity were studied. Grading of severity and management were done following WHO guidelines. Electrocardiogram, 2D echocardiography and cardiac enzymes assay were done to find out cardiac manifestation. Other major organ involvements were also assessed.

Results: Of the 50 patients, 32(64\%) were males and 18(36\%) were female. The mean age of the patients was $30 \pm 8.5$ years. The commonest clinical presentation was fever (94\%). On clinical examination $54 \%$ of the patients had petecheia. Rhythm disturbance was noted in $8 \%$ of the patients. with AV block being the most common (66.67\%). Cardiac manifestation in the form of changes in ECG and echocardiography were observed in $52 \%$ of the patients with a positive correlation with the severity of the dengue fever as defined by W.H.O. criteria. Cardiac manifestation was also found to be significantly related to other organ involvement. Conclusion: Patients with dengue fever are at high risk of developing cardiac involvement and therefore require a close cardiac monitoring.
\end{abstract}

\section{Introduction}

Dengue fever (DF) and its severe forms dengue haemorrhagic fever (DHF) and dengue shock syndrome (DSS) have become major international public health concerns. Over the past three decades, there has been a dramatic global increase in the frequency of dengue fever (DF), DHF and DSS and their epidemics, with a concomitant increase in disease incidence. Dengue is found in tropical and subtropical regions around the world, predominantly in urban and semi-urban areas. The name dengue is derived from the Swahili word ' $\mathrm{ki}$ denga pepo' which means 'sudden seizure by the demon'. The term 'break bone fever' was coined following the Philadelphia epidemic in 1780. The earliest description of dengue fever was made in the same year by Benjamin Rush. The other names for dengue fever are dandy fever, denga, dunga fever and coupd-barre. Humans and mosquitoes are the principal hosts of dengue virus; the mosquito remains infected for life but the viruses are only known to cause illness in humans. There is no specific treatment for dengue, but appropriate medical care frequently saves the lives 
of patients. The first confirmed epidemic of DHF was recorded in the Philippines in 1953-1954 and in Thailand in 1958. Since then, Member countries of the WHO South-East Asia (SEA) and Western Pacific (WP) regions have reported major dengue outbreaks at regular frequencies. In India, first confirmed DHF outbreak occurred in 1963. Due to the high disease burden, dengue has become a priority area for several global organizations other than WHO, including the United Nations Children's Fund (UNICEF), United Nations Environment Programme (UNEP), the World Bank, and the WHO Special Programme for Research and Training in Tropical Diseases (TDR), among others.

The dengue viruses form a distinct complex within the genus Flavivirus based on antigenic and biological characteristics. There are four virus serotypes, which are designated as DENV-1, DENV-2, DENV-3 and DENV-4. Infection with any one serotype confers lifelong immunity to that virus serotype. Although all four serotypes are antigenically similar, they are different enough to elicit cross-protection for only a few months after infection by any one of them. Secondary infection with another serotype or multiple infections with different serotypes leads to severe form of dengue (DHF/DSS). Unusual manifestations of patients with severe organ involvement such as liver, kidneys, brain or heart associated with dengue infection have been increasingly reported in DHF even in dengue patients who do not have evidence of plasma leakage.

Cardiac manifestations in dengue virus infection can range from asymptomatic bradycardia to life threatening myocarditis. Various studies have quoted several cardiac manifestations of dengue infection like sinus bradycardia, transient $\mathrm{AV}$ blocks, ventricular arrhythmias, myocarditis and pericardial effusion. We performed this study with the objective of studying the cardiac manifestations of dengue and to study the correlation of the cardiac manifestations to the severity of dengue.
Objectives of the Study

1. To study the prevalence of cardiac manifestation of dengue patients in our hospital.

2. To find out the correlation of cardiac manifestations to severe forms of dengue; DHF and DSS.

\section{Materials and Methods}

The present study, a two year observational study was conducted in the Department of Medicine, $\mathrm{DMCH}$, bihar on 50 patients presenting with dengue fever from July2014 to July 2016.

\section{Inclusion Criteria}

Age group of $\geq 13$ years fulfilling the WHO criteria for dengue who Confirmed dengue serology (NS1Ag/Ig M antibody for dengue/IgM and $\mathrm{Ig} G$ antibody for dengue)

\section{Exclusion criteria}

- Patients on medications affecting the heart rate / rhythm (calcium channel blocker, B blocker, xanthine derivative, B agonist)

- Patients with history of preexisting heart disease

- Patients with electrolyte abnormalities which may affect the heart rate/rhythm

- Patients not willing to give consent for the study.

- Patients with mixed infection.

Ethical clearance was obtained prior to the study and written informed consent was obtained from the participating patients. The patients underwent general physical examination, systemic examination and a group of tests that included: Complete blood count, Platelet count, liver function test, renal function test, electrocardiography, 2D Echocardiography, cardiac enzymes Troponin $\mathrm{T}$ and Troponin I test by commercial ELISA kit available. Serial ECGs were taken on everyday during hospital stay. All the patients were evaluated using two-dimensional echocardiography on day one and day three, and day seven or day of discharge(whichever is earlier). Dengue patients positive for one of the following investigations with or without clinical features 
were considered as cardiac involvement, features suggestive of rhythm disturbance, heart rate changes like sinus tachycardia or bradycardia, and abnormal ECG and ECHO. Echocardiography changes were interpreted as; Systolic dysfunction - Refers to impaired ventricular contraction. Diastolic dysfunction - Refers to an abnormality in the filling during diastole. Ejection fraction The fraction of the blood pumped out of a ventricle with each heart beat (Normal value -67 $\pm 12 \%$ ) Pericardial effusion- An abnormal accumulation of fluid in the pericardial cavity. Additional investigations were done as appropriate if signs suggestive of any other system involvement found in clinical examination.

Severity of Dengue Fever in patients who were seropositive for Dengue were classified as follows:

- Dengue Fever (DF) (without or with unusual bleed)

- Dengue Hemorrhagic Fever (DHF) Four cardinal features of DHF as defined by WHO are as follows

1. F ever or history of fever lasting 2-7 days, occasionally biphasic

2. A hemorrhagic tendency shown by at least one of the following: a positive tourniquet test; petechiae, ecchymoses or purpura; bleeding from the mucosa, gastrointestinal tract, injection sites or other locations; or hematemesis or melena

3. T hrombocytopenia $[\leq 100,000$ cells $/ \mathrm{mm} 3$ $(100 \times 109 / \mathrm{L})]$

4. Evidence of plasma leakage owing to increased vascular permeability shown by: an increase in hematocrit $\geq 20 \%$ above the average for age, sex and population; a decrease in the hematocrit after intervention $\geq 20 \%$ o f baseline; signs of plasma leakage such as pleural effusion, ascites or hypoproteinaemia.

- Dengue Shock Syndrome (DSS) - For a case of DSS, all four criteria for DHF must be met, in addition to evidence o $\mathrm{f}$ circulatory failure manifested as

-Rapid and weak pulse and narrow pulse pressure $(<20 \quad \mathrm{mmHg}$ o $\quad \mathrm{r} \quad 2.7 \mathrm{kPa})$ manifested by Hypotension for age and Cold, clammy skin and restlessness or lethargy.

All patients were managed according to WHO guideline and with intravenous fluid and transfusion as required.

Statistical Analysis: The categorical data was expressed as rates, ratios and proportions and comparison was done using chisquare test and Fisher's exact test. The continuous data was expressed as mean \pm standard deviation (SD).A probability value ('p' value) of less than or equal to 0.05 at $95 \%$ CI was considered as statistically significant.

\section{Results}

The mean age of our study group was $30+/ 8.5$ years. The youngest patient was 13 years old and the oldest patient was 67years old. There were $32(64 \%)$ males and $18(36 \%)$ females in our study. $44(88 \%)$ patients presented to us within the first week of fever. Forty-seven(94\%) were febrile at admission Of the 50 patients studied, 32 patients were dengue NS1 positive (ELISA), 12 were positive for IgM and IgG dengue (ELISA) both, and 6 patients with IgM dengue (ELISA) only. 50 (100\%) patients were with platelet count $<100000 /$ cumm with $28(56 \%)$ patients having platelet count $<20000 /$ cumm. Fever was the most common clinical presentation (94\%) followed by myalgia (76\%) and others. (figure 1) 


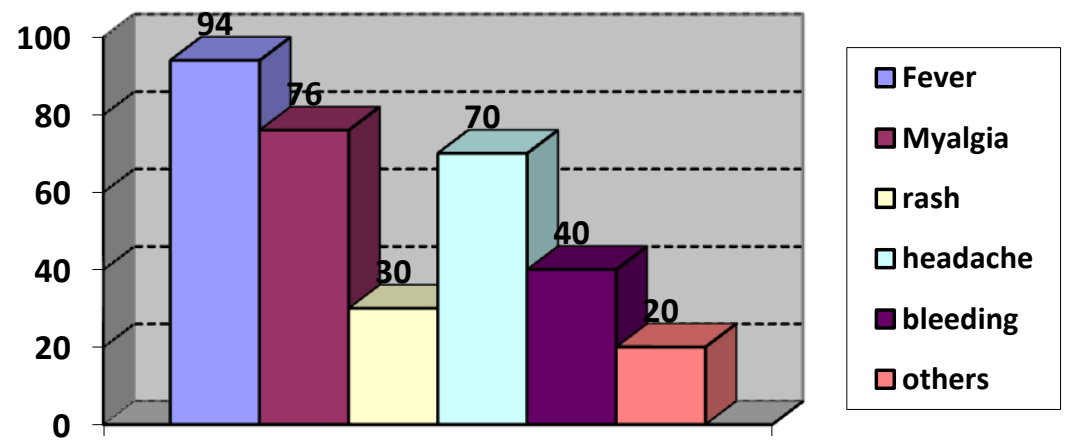

Figure 1

Petechiae was commonest sign (54\%) followed by others. (figure 2) Shock signs includedtachycardia (HR>100 beats/min), narrow pulse pressure $(<30 \mathrm{~mm}$ of $\mathrm{Hg})$, tachypnea $(>20$ cycles/min), low blood pressure and cold peripheries narrow pulse pressure.

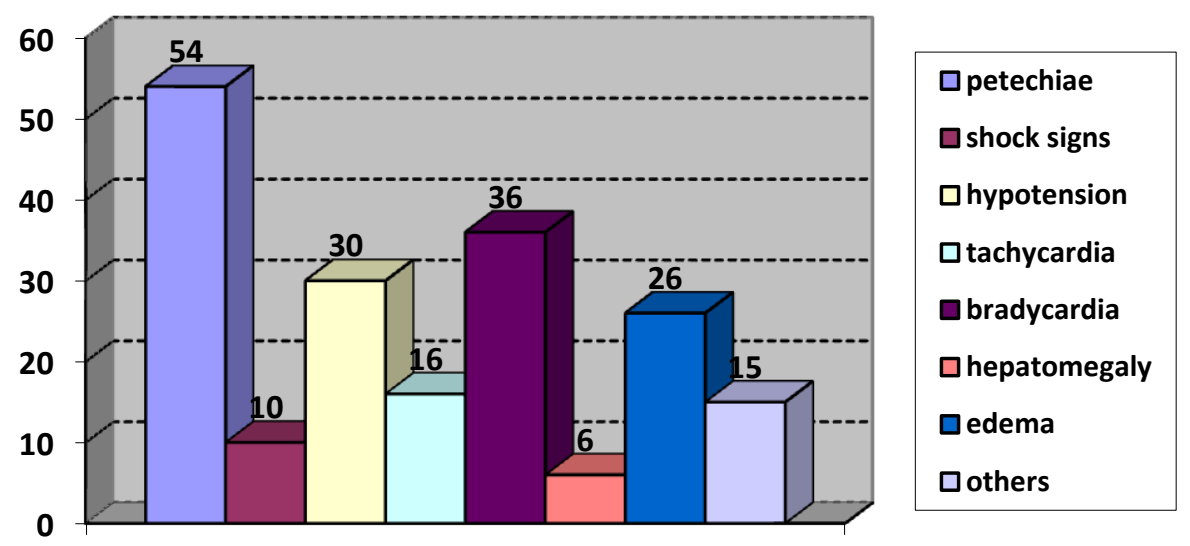

Figure 2

At the time of admission, 30(60\%) patients had dengue fever,15(30\%)had DHF and 5(10\%) had DSS. Based on ECG and echocardiography
26(52\%) patients had cardiac manifestations. Cardiac enzyme assay was done qualitatively (by kit test) and all results were negative.

Table 1: ECG finding at admission (figure:3)

\begin{tabular}{|l|l|c|}
\hline \multirow{2}{*}{$\begin{array}{l}\text { Sinus } \\
\text { rhythm }\end{array}$} & $46(90 \%)$ \\
\cline { 2 - 3 } & Sinus bradycardia & $14(28 \%)$ \\
\cline { 2 - 3 } & Sinus tachycardia & $6(12 \%)$ \\
\cline { 2 - 3 } & ST-Tchanges & $2(4 \%)$ \\
\hline AV block(1 $\left(1^{\text {st }}\right.$ degree $)$ & $3(6 \%)$ \\
\hline AV block(2 $2^{\text {nd }}$ degree $)$ & $1(2 \%)$ \\
\hline
\end{tabular}




\section{ECG findings of patients}

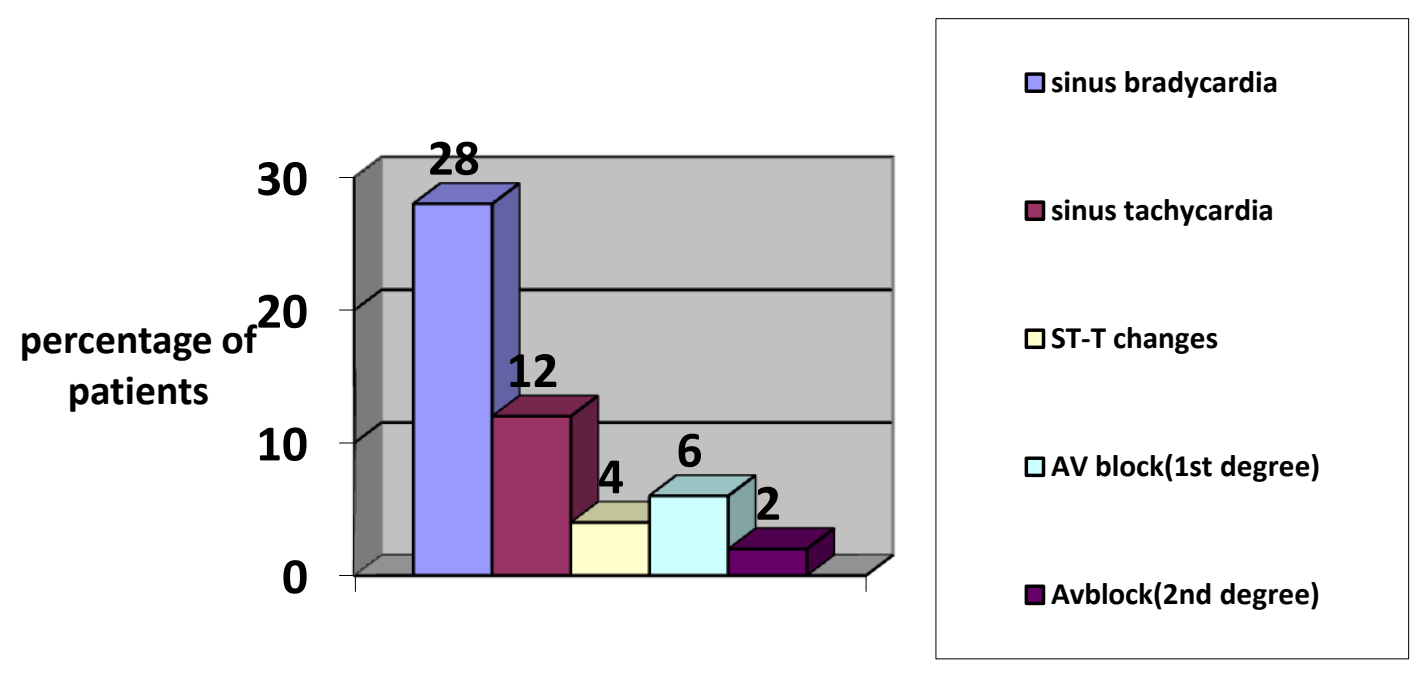

Figure 3

Table 2 : echo cardiographic finding at admission

\begin{tabular}{|l|c|}
\hline Normal & $45(90 \%)$ \\
\hline Reduced LVEF & $3(6 \%)$ \\
\hline Pericardial effusion & $2(4 \%)$ \\
\hline LV hypokinesia & $1(2 \%)$ \\
\hline
\end{tabular}

\section{ECHO findings of patients}

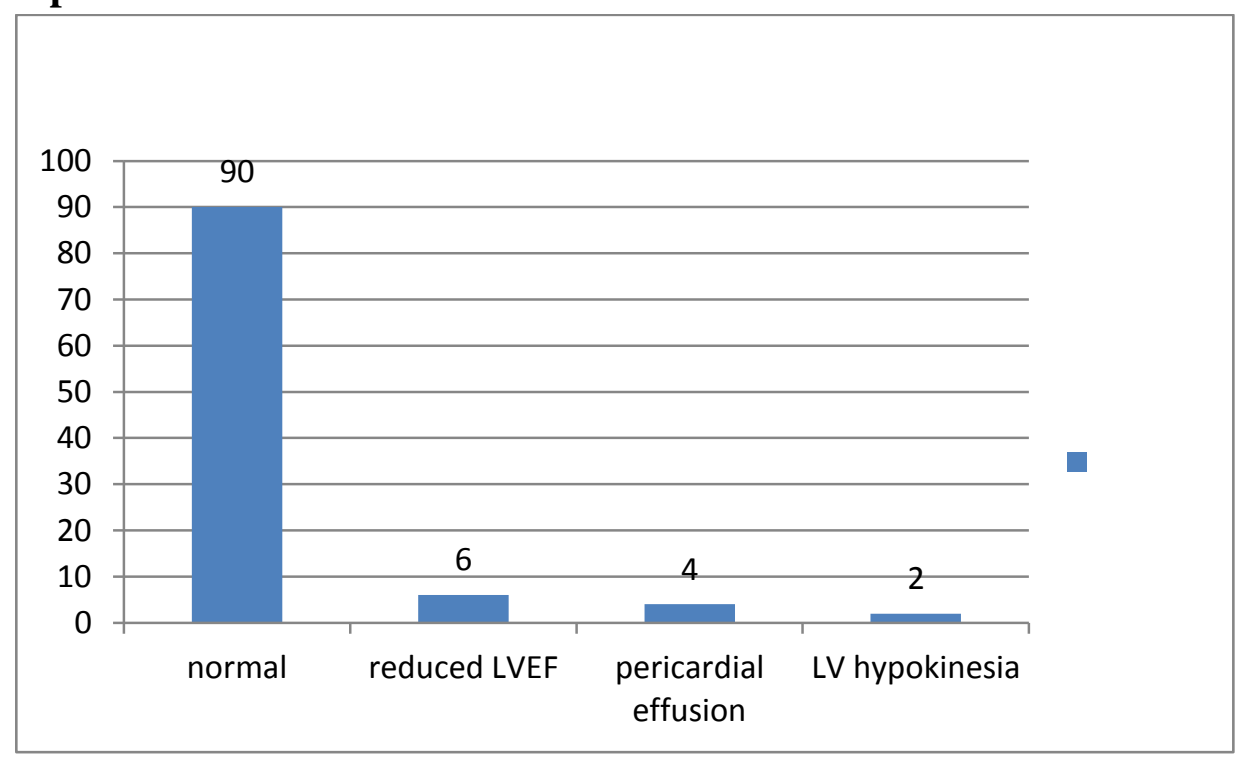

Figure 4

$3^{\text {rd }}$ day onwards all ECG and echocardiography were normal. Hence all cardiac manifestations were transient and reversible.
Other organ involvement also recorded in 20(40\%) patients.(table 3) 
Table: 3

\begin{tabular}{|l|l|l|}
\hline \multirow{4}{*}{$\begin{array}{l}\text { Neurological } \\
\text { involvement }\end{array}$} & \multicolumn{3}{|l|}{$8(16 \%)$} & $7(14 \%)$ \\
\cline { 2 - 3 } & Encephalitis/aseptic meningitis & $(1 \%)$ \\
\cline { 2 - 3 } & Transverse myelitis & $9(18 \%)$ \\
\hline \multirow{5}{*}{ Gastrointestinal } & $15(30 \%)$ & $1(2 \%)$ \\
\cline { 2 - 3 } & Hepatitis & $1(2 \%)$ \\
\cline { 2 - 3 } & Acalculous cholecystitis & $5(10 \%)$ \\
\cline { 2 - 3 } & Pancreatitis & $7(14 \%)$ \\
\cline { 2 - 3 } & Gastrointestinal bleeding & $5(10 \%)$ \\
\hline \multirow{5}{*}{ Renal } & $12(24 \%)$ & $7(14 \%)$ \\
\cline { 2 - 3 } & Acute kidney injury & $3(6 \%)$ \\
\cline { 2 - 3 } & Haemolytic uremic syndrome \\
\cline { 2 - 3 } & Haematuria & $1(2 \%)$ \\
\cline { 2 - 3 } & $4(8 \%)$ & $4(8 \%)$ \\
\cline { 2 - 3 } & ALI/ARDS & \multicolumn{2}{|l}{} \\
\cline { 2 - 3 } & Pulmonary haemorrhage & \multicolumn{2}{|l}{} \\
\hline Musculoskeletal & $4(8 \%)$ & Myositis with raised CPK \\
\cline { 2 - 3 } & $2(4 \%)$ & \multicolumn{2}{|l}{} \\
\hline Others & \multicolumn{2}{|l}{} \\
\hline
\end{tabular}

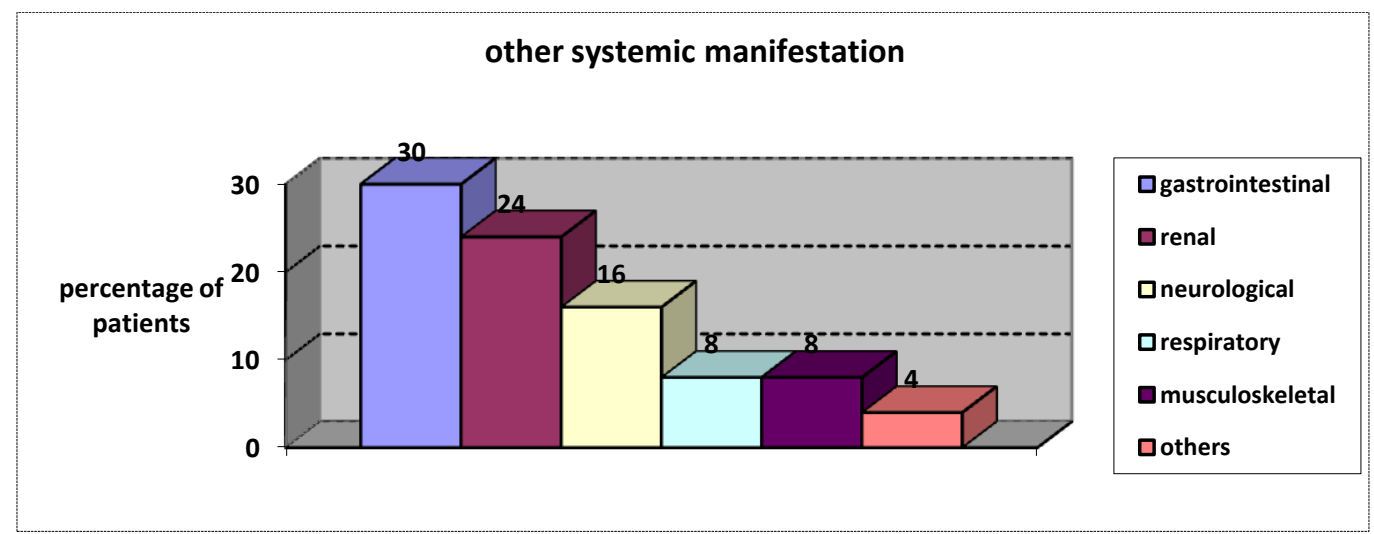

Figure :4

Cardiologic manifestation were significantly higher in DHF and DSS. (table 5) Cardiologic manifestations were also observed to be associated with involvement of other system involvement.(table6)Both results were statistically significant.

\section{Table 5:}

\begin{tabular}{|l|c|c|c|c|}
\hline & $\begin{array}{c}\text { Dengue } \\
\text { fever(n=30) }\end{array}$ & DHF(n=15) & DSS(n=5) & P value \\
\hline ECG abnormality & $10(33.33 \%)$ & $12(80 \%)$ & $4(80 \%)$ & 0.004 \\
\hline $\begin{array}{l}\text { Echocardiographic } \\
\text { abnormality }\end{array}$ & $1(3.33 \%)$ & $2(13.33 \%)$ & $2(40 \%)$ & 0.018 \\
\hline
\end{tabular}

Table 6:

\begin{tabular}{|l|c|c|c|}
\hline $\begin{array}{l}\text { Other organ } \\
\text { involvement }\end{array}$ & $\begin{array}{c}\text { With Cardiac } \\
\text { Manifestation(n=26) }\end{array}$ & $\begin{array}{c}\text { Without cardiac } \\
\text { Manifestation }(\mathrm{n}=24)\end{array}$ & P value \\
\cline { 1 - 3 } Present $(\mathrm{n}=20)$ & 14 & 6 & \multirow{2}{*}{0.03} \\
\cline { 1 - 3 } Absent $(\mathrm{n}=30)$ & 12 & 18 & \\
\hline
\end{tabular}




\section{Discussion}

Dengue is one of the most important emerging viral diseases globally. The majority of symptomatic infections result in a relatively benign disease course. However, a small proportion of patients develop severe clinical manifestations, including bleeding, organ impairment, and endothelial dysfunction with increased capillary permeability causing hypovolemic shock that can lead to cardiovascular collapse. Cardiac dysfunction associated with the acute phase of dengue fever has been under diagnosed in clinical practice. Although cardiac manifestations specific to dengue are rare, depression of myocardial function is frequent in dengue hemorrhagic fever and dengue shock syndrome. Cardiac involvement in dengue fever is often under diagnosed due to low index of clinical suspicion and its overlapping clinical manifestations such as hypotension, tachycardia, pulmonary edema and capillary leak associated with dengue virus infection.

The mean pulse rate of the study population was $66 /$ minute. The minimum pulse rate was 48/ minute and the maximum pulse rate was 120 /minute with a standard deviation 15.86 . In the study by Latheef et al mean heart rates were significantly lower in the dengue group 87.6 $( \pm 12.5)$ beats/min (dengue) compared to the control group. The commonest rhythm abnormality noted was sinus bradycardia, found in $32 \%$. Three patients had unexplained sinus tachycardia. In the study by Gupta et al, sinus bradycardia was found in $14.28 \%$, and sinus tachycardia in $21.4 \%$ patients. AV dissociation with sinus node dysfunction was observed in one patient, which resolved in 24 hours. Kaushik et al have described atrioventricular dissociation and sino atrial exit block in a child with dengue fever. All these changes reverted back to sinus rhythm in 24 hours. Chuah et al and Veloso et al have described transient ventricular arrhythmias as a cardiac manifestation of dengue fever. Troponin $\mathrm{T}$ testing was done in all patients and it was found to be negative in all.Kularatne et al in their study I $n$
2007 found $62.5 \%$ of 120 adult dengue patients had ECG changes. They divided patients into "Cardiac group" or "Non-cardiac group" based on presence or absence of ECG changes. Higher incidence of tachycardia, bradycardia and hypotension in "Cardiac group"were found with statistical significance.

Satarasinghe et al in 2007 found that $24 \%$ out of 217 patients had echocardiographic abnormality while none had clinical features of overt myocarditis (ssuch as significant sinus tachycardia, raised jugular venous pressure, triple rhythm, bilateral pulmonary crepitations) All patients showed improvement at three months. A study by Carlos Henrique Miranda et al found 2 patients had reduced LV function, two had LV segmental hypokinesia and one had pericardial effusion. In our study $52 \%$ patients showed cardiac manifestation with sinus bradycardia (28\%) commonest. $10 \%$ had echocardiographic evidence of cardiac involvement. In our study both ECG and ECHO findings were reversible and improved before discharge.

It is widely accepted that dengue haemorrhagic fever is an immunologically mediated disease, a mechanism similar to those involved in viral myocarditis. In a study conducted by Wali et al in 1998, 17 patients with DHF/DSS were studied.12 $(70.59 \%)$ patients had global hypokinesia in echocardiography.7patients had an ejection fraction less than $40 \% .8$ patients had DSS with a mean ejection fraction $39.63 \%$,out of which 5 had an ejection fraction less than 40.They opined that acute reversible cardiac insult may be responsible for hypotension or shock in these patients. Though Carlos Henrique Miranda et al found no significant difference of cardiac manifestation in different grades of severity in dengue, they stated two deaths of patients from cardiogenic shock. A study of hemodynamic profiles in DHF from Thailand reported lowered cardiac index due to decreased ejection fraction and lowered preload. The incidence o f cardiac manifestations was stated to be high $(53.33 \%)$ in patients with dengue shock syndrome compared to the patients with 
dengue hemorrhagic fever (35.29\%) and dengue fever (30\%) in study of Mohit Arora, Rekha S Patil in 2016.In our study prevalence of cardiac manifestation was found to be significantly higher in patients with DHF and DSS. Hence an adequate approach to the hemodynamic instability associated with dengue requires not only a significant volume expansion, but also evaluation and treatment of the accompanying ventricular dysfunction.

Dengue may have varied and multisystem presentations. Encephalopathy by Kho et al. (1981), Row et al. (1996), Thakare et al. (1996), Cam et al. (2001); Encephalitis/aseptic meningitis by Lum et al. (1996), Hommel et al. (1998); Intracranial haemorrhages/ thrombosis by Luiz Jose de Souza et al.; Mononeuropathies polyneuropathies/ Guillane-Barre Syndrome by Soares et al. (2006) were reported as neurological manifestation. Gastrointestinal manifestation was reported as Hepatitis/ fulminant hepatic failure by Lawn et al (2003), Acalculous cholecystitis by Sharma et al. (2006), Goh \& Tan (2006), Wu et al. (2003); Acute pancreatitis by Jusuf et al. (1998), Chen et al. (2004); Febrile diarrhea Helbok et al. (2004); Acute parotitis Torres et al. (2000)Renal involvement as Hemolytic uremic syndrome by Wiersinga et al(2006); Renal failure by Hommel et al (1999), Wiwanitkit (2005a,b);pulmonary involvement as ARDSby Sen et al. (1999), Thong (1998), Lum et al. (1995) Pulmonary hemorrhage by Setlik et al. (2004), Liam et al. (1993); Musculoskeletal involvement as Myositis by Kalita et al. (2005) Rhabdomyolysis by Gunasekera et al. (2000), Davis \& Bourke (2004) were reported. Multisystem involvement also noted in our study and interestingly had a significant association with cardiac manifestation. Not all cases of myocardial dysfunction are due to direct or indirect myocardial structural damage. Possible reasons include Plasma leakage, Cytokine response associated with the infection (TNF- $\alpha$ and IL -6, IL -13, IL -18)as stated by Hober et al in 1993. The endothelium is the target of the immunopathological mechanisms in dengue and DHF. The hallmark is vascular permeability and coagulation disorders. These mechanisms can explain varied systemic involvement. Further studies are needed to understand the interrelation of different system involvement manifestations.

\section{Limitation}

1) Quantitative assay of cardiac biomarkes were not done in this study.

2) Corelation of cardiac manifestation with biochemical marker liked platelet count was not done.

3) Difference of cardiac manifestation between primary and secondary infection of dengue were not assessed.

4) central venous pressure was not measured or correlated with cardiac manifestation.

5) Correlation of clinical finding and investigational findings were not assessed.

\section{Conclusion}

Simultaneous evaluation of all included patients with biomarkers, ECG and 2D-echo was done on a reasonable sample size in the present study. All the patients were followed till the day of discharge and rate and rhythm reverted back to normal in all the patients suggesting transient reversible cardiac abnormality. Each patient were stable with treatment and discharged home finally. Transient cardiac abnormality can be an important presentation and this should guide the treating physician to look for cardiac involvement. There was statistically significant correlation between cardiac manifestations and severe forms of dengue, i.e. DHF and DSS. Hence, awareness regarding cardiac manifestation in dengue can help in the early recognition of dengue complications and reduction of death associated with dengue virus infection.

\section{Abbreviation}

ECG=electrocardiogram, ELISA=Enzyme linked immune sorbent assay; $\mathrm{DHF}=$ Dengue haemorrhagic fever, DSS=Dengue shock 
syndrome; A-V=atrio ventricular; $\mathrm{NS} 1=$ non structural protein 1

\section{References}

1. Chuah SK.Transient ventricular arrhythmia as a cardiac manifestation in dengue haemorrhagic fever--a case report. Singapore Med J 1987; 28:569-72.

2. Clinical and epidemiological characterisation of dengue haemorrhagic fever in Neiva, Colombia, 2004. Rev Salud Publica (Bogota) 2007; 9:53-63.

3. Daniel R, Rajamohanan et al: A Study of Clinical Profile of Dengue Fever in Kollam, Kerala, India. Dengue Bulletin 2005; 29

4. Gupta V, Gadpayle et al:Subclinical Cardiac Involvement in Dengue Haemorrhagic Fever. IACM 2010; 11:107-11.

5. Horta Veloso H, Ferreira Júnior JA, Braga de Paiva JM, et al. Acute atrial fibrillation during dengue hemorrhagic fever. Braz $\mathbf{J}$ Infect Dis 2003; 7:418-22.

6. J M Pelupessy E R Allo,S Jota; Pericardial effusion in dengue haemorrhagic fever. Paediatrica Indonesiana 1989; 29:72-5.

7. K abra S K, Verm a, e t al . Denguje haemorrhagic fever in children in Delhi Bulletin of the World Health Organization 1992; 70:105-108.

8. Kaushik JS, Gupta P, Rajpal S, Bhatt S. Spontaneous resolution of sinoatrial exit block and atrioventricular dissociation in a child with dengue fever. Singapore Med J 2010; 51:e146-8.

9. Khongphathanayothin A, Suesaowalak M, Muangmingsook S, Bhattarakosol $\mathrm{P}$, Pancharoen C. Hemodynamic profiles of patients with dengue hemorrhagic fever during toxic stage: an echocardiographic study. Intensive Care Med 2003; 29(4): 570574.
10. Lateef A, Fisher D et al: Dengue and Relative Bradycardia. Emerging Infectious Diseases 2007; 13:651.

11. Mohit Arora, Rekha S Patil. Cardiac Manifestation in Dengue Fever.Journal of The Association of Physicians of India Vol. 64 July 2016

12. Miranda $\mathrm{CH}$, Borges Mde $\mathrm{C}$, Matsuno $\mathrm{AK}$, Vilar FC, Gali LG, Volpe GJ, http://www.ncbi.

nlm.nih.gov/pubmed?term=Schmidt A\% 5B Author\% 5D\&cauthor=true\&cauthor_ uid=23784923 et al. Evaluation of cardiac involvement during dengue viral infection. Clin Infect Dis 2013; 57(6):812-9.

13. Nagaratnam N, Siripala K, de Silva N. Arbovirus (dengue type) as a cause of acute myocarditis and pericarditis. $\mathrm{Br}$ Heart J 1973; 35:204-6.

14. Obeyesekere I, Hermon Y. Arbovirus heart disease: Myocarditis and cardiomyopathy following dengue and chikungunya fever-a follow-up study. Am Heart J 1973; 85:186-188,

15. Ramesh S. S, Basavaraju M. M, Sandeep R. Sharma, Shetty Shivakumar, Srinivasa M, Surakshith T. K, Ravichethan Kumar. "Study of Bradycardia in Dengue Fever". Journal of Evolution of Medical and Dental Sciences 2014; Vol. 3, Issue 09, March 3; Page: 2378-2388, DOI: 10.14260/jemds/2014/2148

16. Sam S-S, Omar SFS, Teoh B-T, Abd-Jamil ,J AbuBak ar S. Review of Dengue Hemorrhagic Fever Fatal Cases Seen Among Adults: A Retrospective Study. PLoS Negl Trop Dis 2013; 7:e2194.

17. Sameer Gulati and Anu Maheshwari. Atypical manifestation of dengue. Tropical Medicine and International Health.vol12 no 9pp1087-1095sept 2007

18. Satarasinghe et al. Br J Cardiol. 2007 Jun;14(3):171-3 
19. Shah I. Dengue presenting as viral myocarditis. Dengue Bull 2007; 31:172-173

20. Sheetal S,Elizabeth J.A study on th Cardiac Manifestation of Dengue. Journal of the association of physicians of India.2016;64:30-34

21. TDR/WHO. In Dengue: Guidelines for Diagnosis, Treatment, Prevention and Control. Geneva, Switzerland: World Health Organization; 2009.

22. Thein, Leo et al. Risk Factors for Fatality among Confirmed Adult Dengue Inpatients in Singapore: A Matched Case-Control Study ;DOI: 10.1371/journal.pone.0081060

23. Wali JP, Biswas A, Chandra S. Cardiac involvement in dengue hemorrhagic fever. Intl J Cardiol 1998; 64(1): 31-36.

24. Wichmann, Kularatne et al. Cardiac 34 Journal of The Association of Physicians of India Vol. 64 May 2016 involvement in dengue virus infections during the 2004/2005 dengue fever season in Sri lanka: Southeast Asian J Trop Med Public Health 2009; 40:727-30.

25. Wiwanikit; Dengue Cardiac Infection, A Brief Review; Acta Cardiol Sin 2008;24:

26. World Health Organization. Dengue guidelines for diagnosis, treatment, prevention and control. Geneva: World Health Organization; 2009.

27. Yacoub S, Wertheim H, Simmons CP, Screaton G, Wills B. Cardiovascular manifestations of the emerging dengue pandemic. Nat Rev Cardiol 2014; 11:335-45. 